Application of Gaseous Sphere Injection Method for Modeling Under-expanded H2 Injection

R. Whitesides, R. P. Hessel, D. L. Flowers, S. M. Aceves

December 10, 2010

Combustion Theory and Modelling 
This document was prepared as an account of work sponsored by an agency of the United States government. Neither the United States government nor Lawrence Livermore National Security, LLC, nor any of their employees makes any warranty, expressed or implied, or assumes any legal liability or responsibility for the accuracy, completeness, or usefulness of any information, apparatus, product, or process disclosed, or represents that its use would not infringe privately owned rights. Reference herein to any specific commercial product, process, or service by trade name, trademark, manufacturer, or otherwise does not necessarily constitute or imply its endorsement, recommendation, or favoring by the United States government or Lawrence Livermore National Security, LLC. The views and opinions of authors expressed herein do not necessarily state or reflect those of the United States government or Lawrence Livermore National Security, LLC, and shall not be used for advertising or product endorsement purposes. 


\section{Application of Gaseous Sphere Injection Method for Modeling Under-expanded $\mathrm{H}_{2}$ Injection}

Russell Whitesides ${ }^{\mathrm{a},{ }^{,}}$, Randy P. Hessel ${ }^{\mathrm{b}}$, Daniel L. Flowers ${ }^{\mathrm{a}}$, and Salvador M. Aceves ${ }^{\mathrm{a}}$

${ }^{a}$ Lawrence Livermore National Laboratory, Livermore, CA USA, ${ }^{b}$ Engine Research Center, University of Wisconsin, Madison, WI USA

*Corresponding author. E-mail: whitesides1@1ln1.gov 


\title{
Application of Gaseous Sphere Injection Method for Modeling Under-expanded $\mathrm{H}_{2}$ Injection
}

\begin{abstract}
A methodology for modeling gaseous injection has been refined and applied to recent experimental data from the literature. This approach uses a discrete phase analogy to handle gaseous injection, allowing for addition of gaseous injection to a CFD grid without needing to resolve the injector nozzle. This paper focuses on model testing to provide the basis for simulation of hydrogen direct injected internal combustion engines. The model has been updated to be more applicable to full engine simulations, and shows good agreement with experiments for jet penetration and time-dependent axial mass fraction, while available radial mass fraction data is less well predicted.
\end{abstract}

Keywords: gaseous injection; hydrogen; $\mathrm{H}_{2} \mathrm{ICE}$; gas jets; engines

\section{Introduction}

Hydrogen $\left(\mathrm{H}_{2}\right)$ is a candidate primary energy carrier for future mobile applications [14]. For transportation, the two most likely strategies for conversion from chemical energy to mechanical propulsion are fuel cells and internal combustion engines (ICE). While fuel cells promise high conversion efficiency with no harmful emissions, the technology has thus far been limited by high cost and low durability. On the other hand, ICE have been developed for more than a century and are built in large numbers with low cost and high durability. In addition, modern hydrogen ICE have high energy conversion efficiencies potentially approaching that of fuel cells, especially considering vehicular drive cycles. The main drawback of hydrogen-fuelled ICE $\left(\mathrm{H}_{2} \mathrm{ICE}\right)$ compared to fuel cells is the production of $\mathrm{NO}_{\mathrm{x}}$. But $\mathrm{NO}_{\mathrm{x}}$ can be mitigated by appropriate engine operating strategy (e.g. ultra lean burn) or by aftertreatment. At the very least, $\mathrm{H}_{2} \mathrm{ICE}$ can act as a "bridge" technology until fuel cells become more practical for personal transport. Much more information on $\mathrm{H}_{2} \mathrm{ICE}$ research and technology is available in review papers by White, Steeper, and Lutz [5] and by Verhelst and co-workers [6,7]. 
One of the key design parameters for $\mathrm{H}_{2} \mathrm{ICE}$ is the method of introducing the fuel to the combustion chamber. The two most common methods are port-fuel injection (PFI) and direct injection (DI). Hydrogen PFI has certain advantages: durable low-cost hydrogen PFI are already commercially available and an engine can be fitted for hydrogen PFI without having to modify the combustion chamber. But these advantages of hydrogen PFI may be offset by significant drawbacks. First, hydrogen PFI is especially susceptible to pre-ignition and flashback, when combustible mixture is ignited by hotspots in the chamber and propagates back to the intake manifold, with potential to damage the engine. While pre-ignition and flashback are of concern, these uncontrolled ignition events can be mitigated with a proper injection and valve timing strategy [6]. A more fundamental shortcoming of PFI is the loss of power density, compared to an identical engine operated on gasoline, due to the significant displacement of intake air by the hydrogen [7]. Use of hydrogen DI is attractive as it removes the uncontrolled ignition and power density issues, but requires more substantial changes to the combustion chamber and head design, and requires fuel injectors to operate at significantly higher pressures and temperatures while retaining high reliability. Design of $\mathrm{DI} \mathrm{H}_{2} \mathrm{ICE}$ engines can be greatly improved if predictive modeling can guide toward optimum parameters for engine performance (i.e. efficiency, power density, emissions). For DI, accurate modeling of the hydrogen injection event is vital to overall model predictions of the fuel-air mixing and combustion processes. Much work has gone into the modeling of gaseous jets [8-14], with interest in developing accurate models that do not require resolution of the injector nozzle hole $[8,9]$.

In this work, we present new modeling refinements and applications for a gaseous sphere injection (GSI) model that was developed previously [8]. The 
previous work investigated the GSI model for air-into-air, methane-into-air, and helium-into-nitrogen injections at fully expanded and moderately under-expanded conditions. The new refinements include a more flexible method for identifying the jet boundary, which is used to modify turbulence parameters inside the jet. In addition, the values for the modified turbulence parameters were adjusted for the higher pressure-ratio cases studied. The model is applied to two different sets of experimental data for high pressure-ratio hydrogen gas injection into nitrogen environments, spanning a wide range of conditions representative of $\mathrm{DI} \mathrm{H}_{2} \mathrm{ICE}$ applications.

\section{Computational methods}

\subsection{Model details}

The model employed here was described previously [8] and implemented in the KIVA-3V computational fluid dynamics (CFD) code [15]. The basis for the methodology is a modification of the liquid fuel injection algorithm already incorporated in KIVA. The liquid injection algorithm introduces a distribution of parcels of liquid where each parcel corresponds to a number of liquid droplets of a specified radius and temperature consistent with the fuel and nozzle being modeled. Once in the computational domain, the parcels exchange mass, momentum, and energy with their gaseous surrounding through evaporation, drag, and heat transfer, respectively. The liquid injection model has the computationally advantageous characteristics that it does not require nozzles to be placed at computational boundaries and it does not require fine elements around the nozzle location, as it is not necessary to resolve the flow at the nozzle exit. 
Adapting the liquid injection model for gaseous injection allows one to simulate under-expanded jets in complex geometries without the need for very fine mesh resolution. For example, examining the effect of nozzle orientation in asymmetric engine geometries generally requires generating grids for each configuration that resolve the nozzle. This is a cumbersome task that leads to grids with millions of elements $[13,14]$. For the GSI model, the modifications from the liquid fuel injection consist primarily of changing the properties of the injected parcels from those of the liquid fuel to those of the gas as it is injected into the computational domain. Mixing between the injected gas parcels and the surrounding gas is suppressed until the parcels reach a user-specified distance downstream from the nozzle, $X_{\text {core }}$. This parameterization accounts for the existence of an inviscid core described in experiments [16].

In the computational domain, two distinct regions of the gas plume are defined: the core and the jet. Inside these regions the standard RNG k- $\varepsilon$ model is overridden by altering the turbulent intensity, $u^{\prime}$, and the turbulence length scale, $l_{\text {turb }}$, which accounts for the modification of flow turbulence characteristics by the presence of the jet. The standard turbulence model over-predicts gas jet diffusion and underpredicts penetration due to the assumption of isotropic turbulence which is invalid for the highly directional jets [10]. The updates to the model used in the present work consist of changing how the jet region is defined and the value that is used for the turbulence intensity in the jet region.

In the original GSI model [8] the jet region was defined with respect to the injector axis. A computational cell was defined as being in the jet region if:

- the cell was beyond the core region along the injector axis $\left(X \geq X_{\text {core }}\right)$;

- the cell was within twice the steady-state-jet half-radius, $r_{1 / 2}$, (Equation 1 below; from [16]) as measured perpendicular to the injector axis; 


$$
r_{1 / 2}=1.18\left(1.63 \times 0.043 \times\left(X-X_{\text {core }}\right)+\frac{d_{i n j}}{2}\right)
$$

- the cell velocity, $U$, was at least $1 \%$ of the on-axis, steady-state velocity, $\mathrm{u}_{0}$, at that cell's axial distance (Equation 2 below; also from [16]),

$$
u_{0}=1.18 \frac{d_{i n j} / 2}{r_{1 / 2}} v_{i n j}
$$

where $d_{i n j}$ is the injection diameter and $v_{i n j}$ is the injection velocity, which are both discussed in more detail in Section 2.3. This region definition was adequate for the quiescent flows of constant volume injection experiments previously modeled [8], but will inappropriately include regions not influenced by the injection in cases that have otherwise forced flows, such as those in piston-cylinder devices.

We augment the GSI model by setting a further requirement for a computational cell to be considered to be in the jet: the mass fraction of the injected gas must be above a specified threshold value in the computational cell. We have used a mass fraction threshold of 0.01 for the cases currently modeled. Further, the algorithm has been altered from the original model [8] so that the turbulence parameters are only changed during the injection event. The new and old jet region definitions produce approximately the same results in the constant-volume, quiescentenvironment simulations reported here, however future applications of the model include injection into non-quiescent flow fields, e.g. an engine combustion chamber, where the velocity cutoff criterion is not sufficient to designate the region being affected by the jet.

In the GSI model, the turbulence intensity, ${ }^{u_{j e t}}$, in the jet region is specified to be proportional to the ensemble averaged velocity in that cell, $U$ :

$$
u_{j e t}^{\prime}=\phi_{t} U
$$


In the previous work [8], the turbulence intensity ratio, $\phi_{t}$, was set to 0.2 , set based on the air-into-air experimental results of Witze [16]. We found that this 0.2 turbulence intensity ratio did not correctly predict the spreading of the jet for the current experimental comparisons. For the high-pressure ratio experiments modeled in the present work, a value for the turbulence intensity ratio of 0.3 was found to give the best agreement with experimental data through trial and error.

\subsection{Experimental data}

In the original paper describing the GSI model [8], the authors validated the model by simulating a number of gas injection experiments. These experiments ranged from fully expanded (sub-sonic) to moderately under-expanded, with the most underexpanded experiment having a pressure ratio of 6.7. Injected gases included air into air, methane $\left(\mathrm{CH}_{4}\right)$ into air, and helium into nitrogen. In the present work, the focus is on experimental data of hydrogen injections at high-pressure ratios similar to those expected for direct injection into an ICE.

The first cases we examine with the refined GSI model are those from Peterson [17] of $\mathrm{H}_{2}$ injection into nitrogen $\left(\mathrm{N}_{2}\right)$ with ranging pressure ratios from 5.4 to 30.9. Peterson acquired schlieren images of the injection events and computed penetration distances. A $0.80 \mathrm{~mm}$ hole-diameter, three-hole injector nozzle was used in these cases. The chamber density was set to $3.87 \mathrm{~kg} / \mathrm{m}^{3}$, which corresponds to a pressure of $0.336 \mathrm{MPa}$ and injected hydrogen stagnation pressure was varied with values of 1.8, 3.6, 5.2, and 10.4 MPa. In addition, Peterson measured the discharge coefficient of the nozzle as a function of pressure ratio and found it to vary approximately as: 


$$
C_{d}=0.19+0.00033\left(P_{n} / P_{c h}\right)
$$

where $P_{c h}$ is the chamber pressure and $P_{n}$ is the pressure at the nozzle exit, calculated assuming the flow is choked at the nozzle and the expansion from stagnation is isentropic [17].

The second set of data we simulate comes from more recent measurements by Shudo and Oba, who used both schlieren photography and laser induced breakdown spectroscopy (LIBS) to characterize injection of $\mathrm{H}_{2}$ into a constant volume chamber filled with $\mathrm{N}_{2}$ [18]. The LIBS technique was used to measure species composition at points in the jet once calibrated for the experimental apparatus. Shudo and Oba were interested in stratified charge combustion and investigated how mixture distribution changed as the injection event was split into two segments of varying duration. A pressure ratio of 10 was used in all cases $(0.5 \mathrm{MPa}$ in the chamber and $5 \mathrm{MPa}$ injector stagnation pressure). The experiments used a nozzle with $2 \mathrm{~mm}$ hole diameter. The four test cases were single injection of $1 \mathrm{~ms}$, and split injections of $0.3 / 0.7,0.5 / 0.5$, and $0.7 / 0.3 \mathrm{~ms}$. The split injection cases had a $1 \mathrm{~ms}$ interval between pulses. The mass injected in each experiment was $5.6 \times 10^{-4} \mathrm{~g}$ (personal comm.). This amount corresponds to a discharge coefficient of 0.058 .

\subsection{Modeling parameters}

Modeling parameters must be chosen for the application of this model, and in investigating these experiments we seek to apply a common strategy and rationale for setting the various parameters and avoid tuning the model on a case-by-case basis. The goal is to minimize the free parameters to be varied, not only in the present work where the conditions are well characterized, but also for future efforts where less data is likely to be available to determine optimum parameters. 
The gas properties at injection are determined with the pseudo-mach disk assumption [19]. Following this assumption, nozzle exit conditions (denoted by subscript noz) are calculated assuming that flow is choked at the nozzle exit and the expansion process occurs isentropically. The gas properties at the mach-disk (denoted by the subscript inj) downstream of the physical nozzle exit can then be calculated. $P_{i n j}$ is set equal to $P_{c h}, T_{i n j}$ is set equal to $T_{n o z}$ (calculated assuming choked flow at the nozzle and isentropic expansion), $\rho_{i n j}$ is calculated from the ideal gas law, $v_{i n j}$ corresponds to sonic flow at $T_{i n j}$, and the injection diameter, $d_{i n j}$, is calculated from mass conservation as

$$
d_{i n j}=2 \sqrt{\frac{\dot{m}_{n o z}}{\pi \rho_{i n j} v_{i n j}}}
$$

The mass flow rate at the nozzle, $\dot{m}_{n o z}$, must be known or determined, either provided from the experimental data or calculated, usually based on temperature and pressure ratio and a known discharge coefficient for the nozzle, $C_{d}$.

With the above quantities set, the remaining parameters that must be determined are the core length, $X_{\text {core }}$, and the turbulence intensity ratio, $\phi_{t}$, both described above. A larger value of $X_{\text {core }}$ primarily leads to a longer jet penetration length while higher $\phi_{t}$ results in an increased jet-spreading rate. These two parameters are the most difficult to assign based on experimental criteria at the same time allowing for tuning of the model results and so were adjusted in the simulations to provide the best fit to the available data. Other parameters required as part of the GSI model specification are the gas particle Sauter mean radius (SMR), number of parcels, and injection cone angle. These parameters are inherited from the liquid injection model and were kept constant in the reported simulations with values of $10^{-5} \mathrm{~m}, 10^{4}$, and $10^{\circ}$, respectively. Simulation results with SMR, number of parcels, 
and cone angle varied did not deviate significantly from those using the nominal values.

A summary of experimental and modeling parameters for both sets of experiments are given in Table 1. Note that the Shudo and Oba cases are identical except for splitting of the injection event into two pulses, as described above. In addition to the data presented in the table, the modeling of the Shudo and Oba cases assumed that the injection event started $0.3 \mathrm{~ms}$ after the zero time specified by the experimenters. This is motivated by the consistent delay seen in the Shudo and Oba data for both penetration and the axial concentration, and is consistent with the response time of this system.

Figure 1 shows a schematic of the injection event, including hydrogen mass fraction contours, diagrams of $X_{\text {core }}$ and $r_{1 / 2}$, and a cross-section of the upper portion of the numerical grid. The computational domain used in modeling both sets of experimental data consists of a rectilinear volume $40 \times 40 \times 100 \mathrm{~mm}$ composed of 67,500 hexahedral grid elements $(30 \times 30 \times 75)$. The grid spacing was adjusted to create smaller elements near the nozzle with the smallest elements being $\sim 0.9 \mathrm{~mm}$ on a side. The injector was placed centrally in the $x-y$ plane at $z=100 \mathrm{~mm}$ pointing in the negative $\mathrm{z}$ direction. The time step was chosen to be a constant $10^{-6} \mathrm{~s}$. The model results were confirmed to be independent of further grid refinement and time step reduction.

The traditional method of handling gaseous injection is to resolve the nozzle and conduct a fully Eulerian simulation. Thus, for comparison, simulations were also performed using a velocity inflow boundary condition for fully Eulerian flow. These simulations were run to evaluate the performance of the GSI method and were only performed for two Shudo and Oba injection cases: single injection and the $0.3 / 0.7 \mathrm{~ms}$ 
split injection. The Eulerian injection simulations used the same pseudo-mach disc assumption to set the effective nozzle radius, velocity, pressure, temperature, and density of the incoming hydrogen. In addition, the same turbulence parameter adjustment in the core and jet regions was performed as for the GSI simulations. As previously noted, resolution of the nozzle requires the numerical mesh to be much finer. Grid independence required mesh refinement up to 480,500 cells, a factor of seven increase over the resolution necessary for grid independence when using the GSI method. The high number of cells results in higher computational cost and so the fully-Eulerian simulations were ended one millisecond after the end of injection. Comparison between results from employing the Eulerian inflow boundary condition and GSI method is presented in Section 3.3.

\section{Results and discussion}

\subsection{Peterson experiments}

Figure 2 shows the penetration data of Peterson along with the modeling results. The penetration was calculated in the modeling results by evaluating the gradient of the fuel mass fraction and determining the position of the peak gradient that corresponded to the leading edge of the gas jet. Good agreement is apparent for all four cases. In the previous study [8], the recommended value for $X_{\text {core }}$ was given as $6.25 \times d_{i n j}$ based on a correlation from Witze's air into air measurements [16]. Note from Table 1 that the values of $X_{\text {core }}$ that gave best agreement do increase with the pressure ratio and hence $d_{i n j}$, however, they do not follow the same correlation as previously cited $[8,16]$. We expect that the optimal value of $X_{\text {core }}$ will change from injector to injector and will also be somewhat mesh dependent (more so for coarser meshes) and therefore the reader should not attempt to draw broad conclusions from the values obtained here. 
With the encouraging agreement of results, we now move onto modeling the more complete data of Shudo and Oba.

\subsection{Shudo and Oba experiments}

Shudo and Oba measured both penetration using schlieren images and fuel mass fraction in the axial and radial directions using the LIBS technique. As the LIBS technique provides more information about the mixture formation process and is expected to be a more accurate measurement, more consideration was given to matching the LIBS data than the penetration measurements. In Shudo and Oba, data was presented by a defined equivalence ratio; we converted this equivalence ratio to mass fraction for comparison with model computations. Figure 3 shows comparison between experiment and simulation of mass fractions as a function of time at three axial locations $(\mathrm{z}=85 \mathrm{~mm}, 75 \mathrm{~mm}$, and $65 \mathrm{~mm}$ with injector at $\mathrm{z}=100 \mathrm{~mm}$ pointed in the negative $\mathrm{z}$ direction) for the four cases.

The agreement between the simulations and the experimental data is good. For each case at each axial location, the timings of the peaks agree well overall, with some differences that can be observed. The magnitudes of the peaks are generally under-predicted in the single injection case and over-predicted in the split $0.3 / 0.7$ and $0.5 / 0.5$ cases. The least well-captured aspect of the experimental data is the timing and shape of the mass fraction peaks at $65 \mathrm{~mm}$. In the single injection case the peak arrives early in the model and is more square-shaped than the experimental. In the split injection cases the model shows two peaks at $65 \mathrm{~mm}$, one for each of the injection pulses, while the measured data shows only a peak corresponding to the second pulse. 
In addition to the time-dependent axial concentration data, Shudo and Oba also measured the $\mathrm{H}_{2}$ concentration as a function of radial distance from the injector axis at $20 \mathrm{~mm}$ from the injector nozzle $(\mathrm{z}=80 \mathrm{~mm}) 0.5 \mathrm{~ms}$ after the end of injection. The radial data is difficult to match as the axial concentration is rapidly changing just after the end of injection (cf. Figure 3), and the simulation results confirm this, as shown in Figure 4. Little difference is observed between the different simulated cases while experimental measurements show greater sensitivity to the injection strategy. The model results for radial mass fraction are in approximate agreement with the experimental data for the single and the split $0.7 / 0.3$ injection cases. The overlap in the modeling results can be explained by examining the axial concentration data and noting that for each case and for each pulse the mass fractions at 85 and $75 \mathrm{~mm}$ reach steady state before the end of injection. As this is the case, the decay from steady state at both locations and hence in between at $80 \mathrm{~mm}$ will be similar in all four cases. This is apparently not true of the experimental measurements. The shape of the simulated results is consistent with the experimental data, suggesting that further refinements of the model, such as in the turbulence model, may lead to better agreement.

The penetration measurements of Shudo and Oba along with the results of modeling are shown in Figure 5. The model penetration data were generated in the same manner as described for the Peterson cases. The agreement between model and experiment is satisfactory, with the single and split $0.3 / 0.7$ cases having better agreement than the split $0.5 / 0.5$ and the split $0.7 / 0.3$ cases. In general, penetration agrees well, but is somewhat over-predicted by the model, which is consistent with the results from the axial and radial concentration data. 


\subsection{Eulerian injection simulation}

Figure 6 shows the comparison of time-dependent mass fraction data at three axial locations for the first two Shudo and Oba cases for both the Eulerian injection simulation and the GSI simulation method (re-plotted from Figure $3 a$ and $b$ ). Experimental data points have been excluded in Figure 6 to allow tighter axis limits and hence better comparison of the two simulation methods. The results for the single injection case show very good agreement between GSI and the full Eulerian simulation. The split injection simulations agree well but show more disagreement between the GSI and Eulerian simulations than the single injection case. The discrepancies between the simulations of the split injection case are small and the quality of both methods are similar when compared to experimental data (cf. Figure 3b). Jet penetration and radial mass fraction data (not shown) show similar agreement between the two simulation methods. The consistent results indicate that the GSI method correctly reproduces the velocity inflow boundary condition at dramatically reduced computational cost. In this case, one millisecond of simulation time took an average 75 hours of processor time for the Eulerian injection simulation and only one hour for the GSI method.

\section{Conclusions}

Simulations using the gaseous sphere injection (GSI) model have been compared with experimental data approximating the conditions of direct injection of $\mathrm{H}_{2}$ into an internal combustion engine. The model is able to match penetration data from experiments with little adjustment of modeling parameters. Agreement with more detailed concentration data is more challenging. Axial concentration profiles were in reasonable agreement, while the available radial concentration data were less well 
predicted, which may be addressed through further refinement of current turbulence treatment or use of higher order turbulence model. Comparison of the GSI model with simulations performed using an Eulerian velocity inflow boundary condition were in good agreement. Overall, the model is an acceptable approximation for capturing the dynamics of high-pressure hydrogen injection at reduced computational cost, and is readily applicable to hydrogen IC engine simulations.

Acknowledgements. This project is funded by DOE, Office of Vehicle Technologies, Gurpreet Singh and Kevin Stork, Technology Development Managers. This work performed under the auspices of the U.S. Department of Energy by Lawrence Livermore National Laboratory under Contract DE-AC52-07NA27344.

References

[1] J.M. Ogden, Phys. Today 55 (2002) 69.

[2] A. Midilli, M. Ay, I. Dincer, M.A. Rosen, Renewable Sustainable Energy Rev. 9 (2005) 255-271.

[3] L. Barreto, A. Makihira, K. Riahi, Int. J. Hydrogen Energy 28 (2003) 267-284.

[4] G.D. Berry, S.M. Aceves, J. Energy Resour. Technol. 127 (2005) 89-94.

[5] C. White, R. Steeper, A. Lutz, Int. J. Hydrogen Energy 31 (2006) 1292-1305.

[6] S. Verhelst, S. Verstraeten, R. Sierens, SAE Paper No. 2006-01-0430 (2006).

[7] S. Verhelst, T. Wallner, Prog. Energy Combust. Sci. 35 (2009) 490-527.

[8] R.P. Hessel, N. Abani, S.M. Aceves, D.L. Flowers, SAE Paper No. 2006-013265 (2006).

[9] Y. Ra, R.D. Reitz, C.J. Rutland, Z. Han, SAE Paper No. 2005-01-0208 (2005).

[10] P. Ouellette, P.G. Hill, J. Fluids Eng. 122 (2000) 743-752.

[11] N. Abani, R.D. Reitz, Phys. Fluids 19 (2007) 125102-13.

[12] T.G. Drozda, J.C. Oefelein, SAE Paper No. 2008-01-0939 (2008).

[13] V. Knop, A. Benkenida, S. Jay, O. Colin, Int. J. Hydrogen Energy 33 (2008) 5083-5097.

[14] U. Gerke, Numerical Analysis of Mixture Formation and Combustion in a Hydrogen Direct-Injection Internal Combustion Engine, Ph.D. thesis, ETH Zurich, 2007.

[15] A.A. Amsden, KIVA-3V: A Block-Structured KIVA Program for Engines with Vertical or Canted Valves, Report No. LA-13313-MS, Los Alamos National Laboratory, 1997.

[16] P.O. Witze, The impulsively started incompressible turbulent jet, Report No. SAND80-8617, Sandia National Laboratories, 1980.

[17] B. Peterson, Transient High-Pressure Hydrogen Jet Measurements, Masters thesis, University of Wisconsin-Madison, 2006.

[18] T. Shudo, S. Oba, Int. J. Hydrogen Energy 34 (2009) 2488-2493.

[19] P. Ouellette, Direct Injection of Natural Gas for Diesel Engine Fueling, Ph.D. thesis, University of British Colombia, 1996. 
Table 1. Summary of experimental and modeling parameters.

\begin{tabular}{|c|c|c|c|c|c|c|c|c|}
\hline \multirow[b]{2}{*}{ Case } & \multicolumn{4}{|c|}{ Peterson [17] } & \multicolumn{4}{|c|}{ Shudo and Oba [18] } \\
\hline & 1 & 2 & 3 & 4 & 1 & 2 & 3 & 4 \\
\hline$P_{0}[\mathrm{MPa}]$ & 1.8 & 3.6 & 5.2 & 10.4 & \multirow{2}{*}{\multicolumn{4}{|c|}{$\begin{array}{c}5 \\
0.5\end{array}$}} \\
\hline$P_{c h}[\mathrm{MPa}]$ & \multicolumn{4}{|c|}{0.336} & & & & \\
\hline$T_{i n j}[\mathrm{~K}]$ & \multicolumn{4}{|c|}{248.3} & \multicolumn{4}{|c|}{248.3} \\
\hline$\rho_{i n j}\left[\mathrm{~kg} / \mathrm{m}^{3}\right]$ & \multicolumn{4}{|c|}{0.326} & \multicolumn{4}{|c|}{0.484} \\
\hline$v_{i n j}[\mathrm{~m} / \mathrm{s}]$ & \multicolumn{4}{|c|}{1202.2} & \multicolumn{4}{|c|}{1202.2} \\
\hline$d_{i n j}[\mathrm{~mm}]$ & 0.588 & 0.833 & 1.00 & 1.43 & \multirow{2}{*}{\multicolumn{4}{|c|}{$\begin{array}{c}1.11 \\
0.058\end{array}$}} \\
\hline$C_{d}$ & 0.191 & 0.192 & 0.193 & 0.195 & & & & \\
\hline$\dot{m}_{n o z}[\mathrm{~kg} / \mathrm{s}]$ & $1.06 \times 10^{-4}$ & $2.14 \times 10^{-4}$ & $3.10 \times 10^{-4}$ & $6.28 \times 10^{-4}$ & \multicolumn{4}{|c|}{$5.6 \times 10^{-4}$} \\
\hline $\mathrm{t}_{\mathrm{inj}}[\mathrm{ms}]$ & \multirow{2}{*}{\multicolumn{4}{|c|}{$\begin{array}{c}4 \\
0.3\end{array}$}} & 1 & $0.3 / 0.7$ & $0.5 / 0.5$ & $0.7 / 0.3$ \\
\hline & & & & & \multicolumn{4}{|c|}{0.3} \\
\hline$X_{\text {core }}[\mathrm{mm}]$ & 0.0 & 3 & 9.5 & 11 & & & & \\
\hline
\end{tabular}


Figure 1. Schematic of injection simulations: left half shows parameters used for determining core and jet regions; right half shows contours of hydrogen mass fraction for typical injection event as well as jet penetration measurement (See text for detailed explanation of penetration measurement in simulations).

Figure 2. Jet penetration data from Peterson experiments [17] (symbols) compared with simulation results (lines). Numbers correspond to cases detailed in Table 1.

Figure 3. Shudo and Oba axial mass fraction measurements [18] (symbols) compared with simulation results (lines) for (a) single injection, (b) split 0.3/0.7, (c) split 0.5/0.5, and (d) split 0.7/0.3.

Figure 4. Shudo and Oba radial mass fraction measurements at $0.5 \mathrm{~ms}$ after end of injection [18] (symbols) compared with simulation results (lines).

Figure 5. Shudo and Oba penetration measurements [18] (symbols) compared with simulation results (lines) for (a) single injection, (b) split 0.3/0.7, (c) split $0.5 / 0.5$, and (d) split 0.7/0.3.

Figure 6. Hydrogen mass fraction data for simulations of Shudo and Oba [18] (a) single injection case and (b) split $0.3 / 0.7 \mathrm{~ms}$ case using GSI method (thick lines) and Eulerian velocity inflow boundary condition (thin lines). 